\title{
Clinical Influence and Predictors of Pacing-Induced Mechanical Asynchrony in Patients with Normal Cardiac Function with Ventricular Lead Placed in Non-Apical Position
}

\author{
Analysis Based on Intrinsic Conduction Disturbance
}

\author{
Masato Okada, ${ }^{1}$ MD, Kazunori Kashiwase, ${ }^{1}$ MD, Akio Hirata, ${ }_{1}^{1}$ MD, Yasuharu Takeda, ${ }^{1}$ MD, \\ Ryohei Amiya, ${ }^{1} \mathrm{MD}$, Yasunori Ueda, ${ }^{2} \mathrm{MD}$, Yoshiharu Higuchi, ${ }^{1} \mathrm{MD}$ and Yoshio Yasumura, ${ }^{1} \mathrm{MD}$
}

\begin{abstract}
Summary
Right ventricular apical (RVA) pacing often causes left ventricular (LV) mechanical asynchrony, which is enhanced by impaired cardiac contraction and intrinsic conduction abnormality. However, data on patients with normal cardiac function and under RV non-apical (non-RVA) pacing are limited.

We retrospectively investigated 97 consecutive patients with normal ejection fraction who received pacemaker implantation for atrioventricular block with the ventricular lead placed in a non-RVA position. We defined mechanical asynchrony as discoordinate contraction between opposing regions of the LV wall evaluated by echocardiography. Asynchrony was detected in 9 (9\%) patients at baseline and in 38 (39\%) under non-RVA pacing $(P<0.001)$. Asynchrony at baseline was significantly associated with complete left bundle branch block $(\mathrm{CLBBB})$ [odds ratio $(\mathrm{OR})=20.8, P<0.001$ ] . Asynchrony under non-RVA pacing was significantly associated with left anterior fascicular block (LAFB) $(\mathrm{OR}=7.14, P<0.001)$ and CLBBB $(\mathrm{OR}=13.3, P=0.002)$ at baseline. New occurrence of asynchrony was significantly associated with LAFB at baseline (OR $=5.88, P=$ 0.001). During a median follow-up period of 4.8 years, the incidence of device-detected atrial fibrillation (AF) was more frequent in patients who developed asynchrony than in those who did not $(53.3 \%$ versus $27.5 \%$, hazard ratio $=2.17,95 \%$ confidence interval $=1.02-4.61, P=0.03$ ).

In patients with normal cardiac function, LAFB at baseline was significantly associated with new occurrence of mechanical asynchrony under non-RVA pacing. Abnormal contraction had a significant influence on the incidence of device-detected AF.
\end{abstract}

(Int Heart J 2018; 59: 1275-1287)

Key words: Left anterior fascicular block, Left ventricular asynchrony, Right ventricular non-apical pacing

$\mathrm{S}$ everal studies have demonstrated the detrimental effects of right ventricular apical (RVA) pacing on cardiac function. Direct electric stimulation of the right ventricular apex induces an abnormal activation sequence and asynchronous ventricular contraction. ${ }^{1-3)}$ An RV non-apical (non-RVA) position including the septum could be a better alternative pacing site with less left ventricular (LV) asynchrony and better longitudinal function than the RV apex. ${ }^{4 \cdot 6)}$ Several clinical studies have indicated that non-RVA pacing can potentially prevent longterm adverse effects on LV function. ${ }^{7-9)}$

Baseline LV volume, LV ejection fraction (LVEF), intrinsic LV asynchrony, and complete left bundle branch block (CLBBB) during intrinsic rhythm are reported predictors of RVA pacing-induced asynchrony. ${ }^{10-12)}$ However, the predictors of pacing-induced asynchrony under nonRVA pacing have not been examined adequately, especially in patients with normal cardiac function. We therefore investigated the association between intrinsic conduc- tion abnormality at baseline and development of mechanical asynchrony under non-RVA pacing in patients with normal LVEF and LV dimension. The prognostic value of the asynchrony motion was also examined.

\section{Methods}

Study population: This was a single-center, retrospective, observational study based on medical records. Of 161 consecutive patients who underwent their first pacemaker implantation for atrioventricular (AV) block between April 2007 and December 2014 in Osaka Police Hospital, Japan, we excluded 31 who did not undergo echocardiography before or after pacemaker implantation. We also excluded 5 with RVA pacing, 16 with $<80 \%$ cumulative percent ventricular pacing, and 12 with $\geq 60 \mathrm{~mm} \mathrm{LV}$ enddiastolic diameter (LVDd) or $<55 \%$ LVEF, ${ }^{13)}$ because we focused on patients with completely normal cardiac function with non-RVA pacing. The remaining 97 patients

From the ${ }^{1}$ Cardiovascular Division, Osaka Police Hospital, Osaka, Japan and ${ }^{2}$ Cardiovascular Division, Osaka National Hospital, Osaka, Japan.

Address for correspondence: Yasunori Ueda, MD, Division of Cardiovascular, Osaka National Hospital, 2-1-14 Hoenzaka, Chuo-ku, Osaka, Osaka 5400006, Japan. E-mail: yellowplaque@gmail.com

Received for publication November 24, 2017. Revised and accepted February 27, 2018.

Released in advance online on J-STAGE November 5, 2018.

doi: 10.1536/ihj.17-672

All rights reserved by the International Heart Journal Association. 


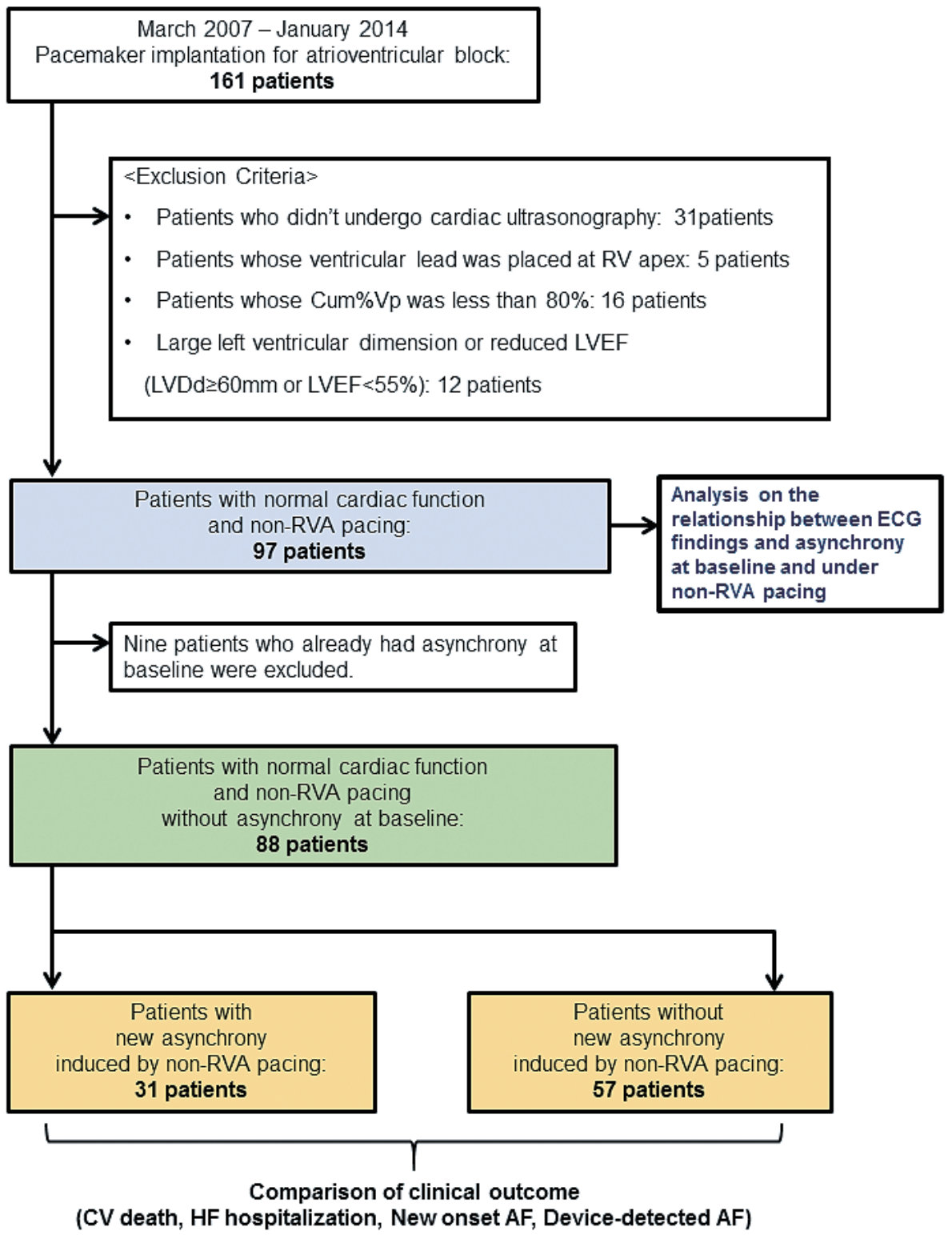

Figure 1. Study flow chart. AF indicates atrial fibrillation; Cum $\%$ Ap, cumulative percent atrial pacing; Cum\% Vp, cumulative percent ventricular pacing; CV, cardiovascular; ECG, electrocardiogram; LVEF, left ventricular ejection fraction; LVDd, left ventricular end-diastolic diameter; and non-RVA pacing, right ventricular non-apical pacing.

were included in the analysis of the relationship between electrocardiographic (ECG) findings and asynchrony motion both at baseline and under non-RVA pacing. Then, additional 9 patients who had asynchrony at baseline were excluded. The remaining 88 patients were divided into 2 groups according to the presence or absence of new asynchrony induced by non-RVA pacing and were compared in terms of clinical outcomes (Figure 1). This study was approved by the ethical committee of Osaka Police Hospital.

Pacemaker implantation and confirmation of pacing site: Since March 2007, we have been primarily trying to place the ventricular lead of the pacemaker in a non-RVA position (i.e., estimated septum). Using a simple curved soft stylet, a ventricular lead delivered into the main pulmonary artery was pulled back with slight counterclockwise rotation and was automatically directed toward the septum. The location of the RV lead was confirmed by conventional fluoroscopic and electrocardiographic criteria. The septal direction of the lead was confirmed by steep left anterior oblique view. Placing the lead in the coronary sinus or in the groove between the septum and anterior free wall was avoided by right anterior oblique view $^{14)}$ (Figure 2A). If the 12-lead electrocardiography results after pacemaker implantation revealed QS pattern in all precordial leads, we excluded the patients because the ventricular lead was located in the RV apex. ${ }^{15)}$ The estimated RV lead position was classified into 3 zones by 
A

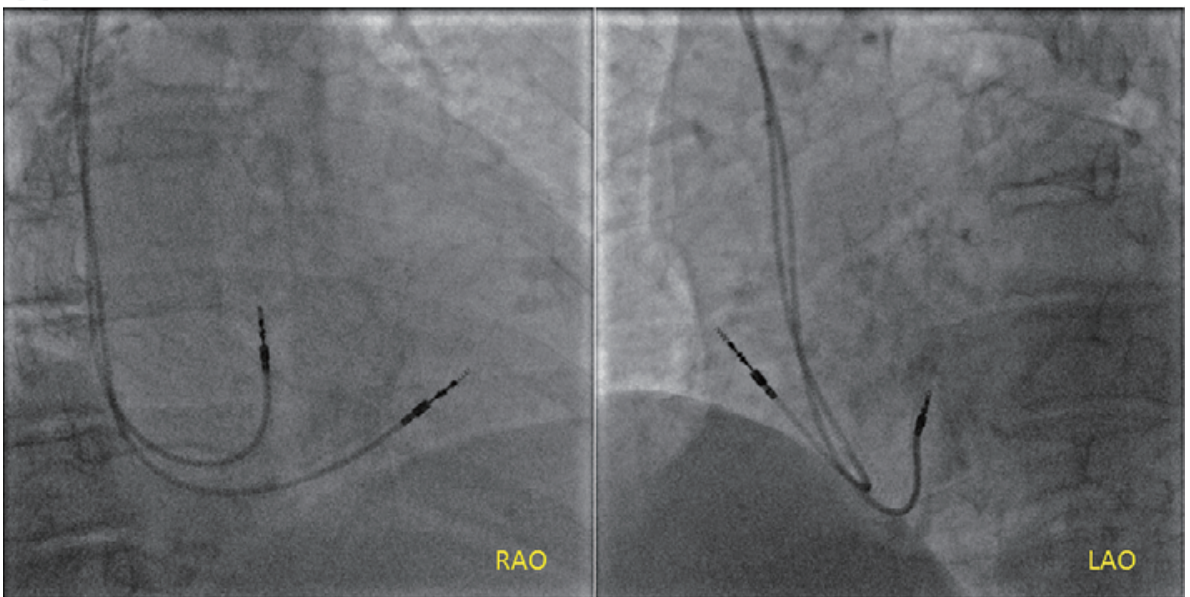

B

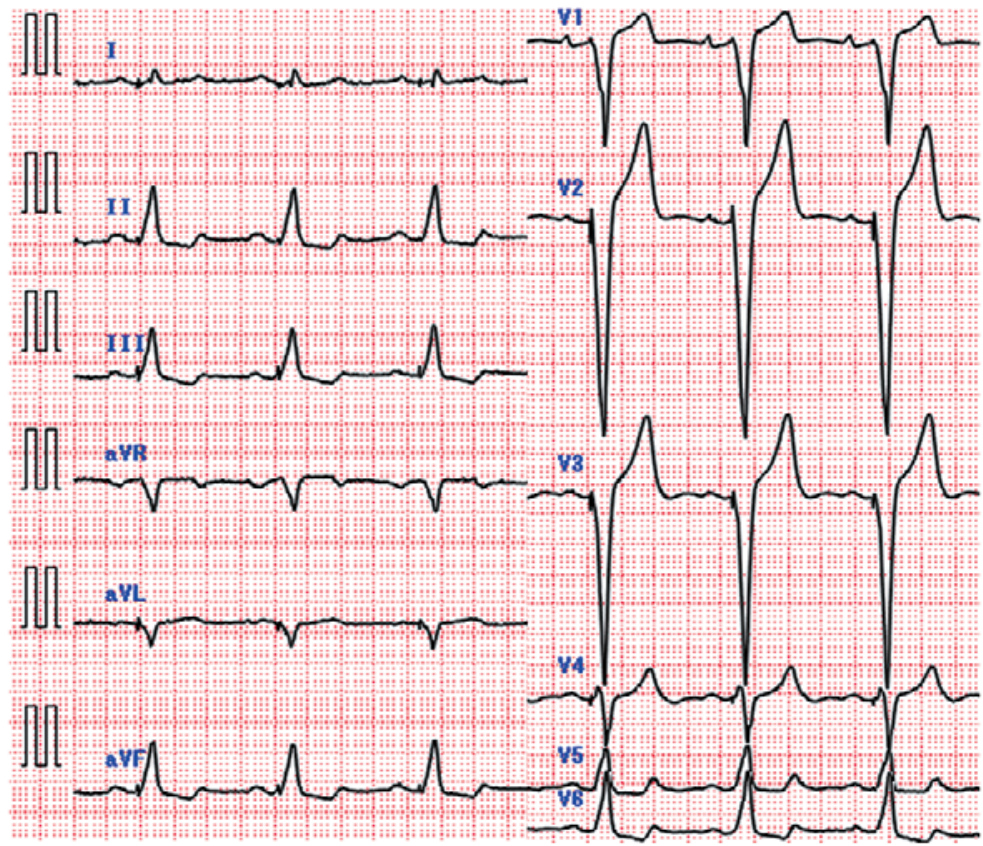

Figure 2. A representative case with the ventricular lead placed in a non-apical position. A: Fluoroscopic image. Left picture shows the image from $30^{\circ}$ right anterior oblique (RAO) view and right picture shows $45^{\circ}$ left anterior oblique (LAO) view. In LAO view, the distal tip of the ventricular lead directed posteriorly indicates the septal position. ${ }^{14}$ ) Additional RAO view was adopted to avoid placing the lead in the right ventricular apex, coronary sinus ostium, or the groove between the septum and anterior free wall. The ventricular lead was estimated to be placed in the RV mid-septum in this patient. B: 12-lead electrocardiography under non-RVA pacing. We tried to place the lead on the right ventricular septum; thus, the transition zone under pacing lies between V4 and V5 in almost all patients in this study. We excluded patients with QS pattern in all precordial leads because the ventricular lead was estimated to be located in the right ventricular apex. ${ }^{15)}$

fluoroscopic images as reported previously: ${ }^{16)}$ 1) highseptum defined as one-third from the top of RV between the pulmonary artery bulge and the roof of the tricuspid valve, 2) mid-septum, and 3) low-septum defined as onethird from the bottom of RV.

Classification based on 12-lead electrocardiography on admission: The 12-lead electrocardiography on the day of admission for pacemaker implantation was used as baseline, and we classified the patients based on the QRS morphology. We evaluated the degree of AV block based on the electrocardiography. In the patients with transient AV block, the degree of AV block was judged by Holter monitoring, implantable loop recorder, or in-hospital monitoring.

For baseline assessment, QRS morphology was classified according to the presence or absence of left anterior fascicular block (LAFB), complete right bundle branch block (CRBBB), or CLBBB (Figure 3). LAFB was de- 


\section{A normal}

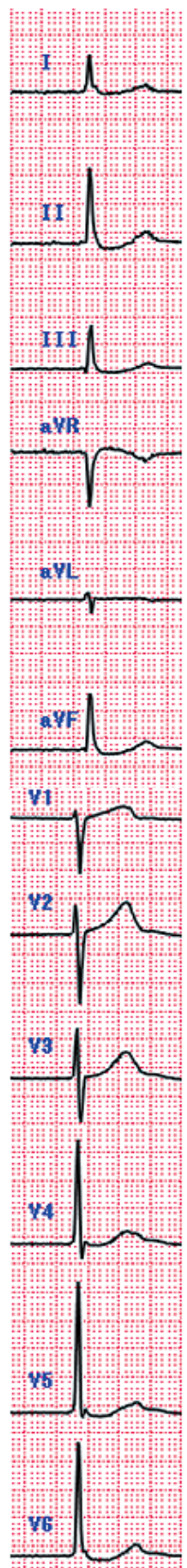

B LAFB

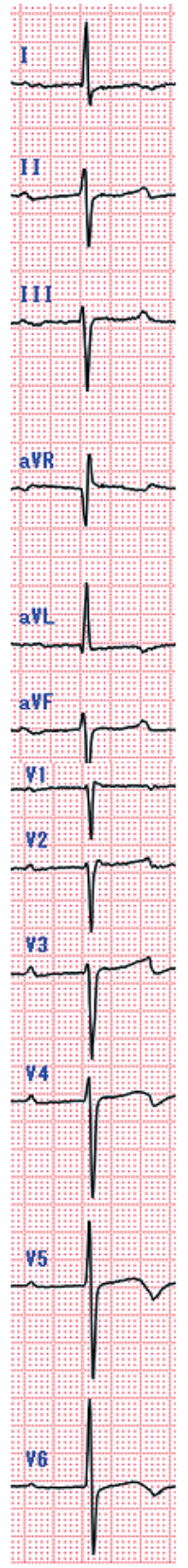

C CRBBB

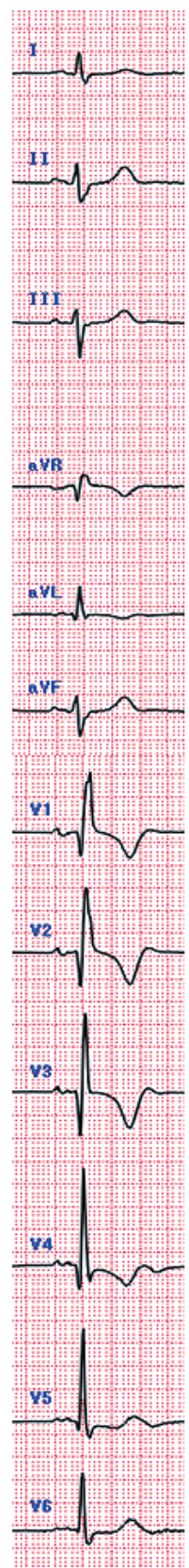

D CRBBB + LAFB

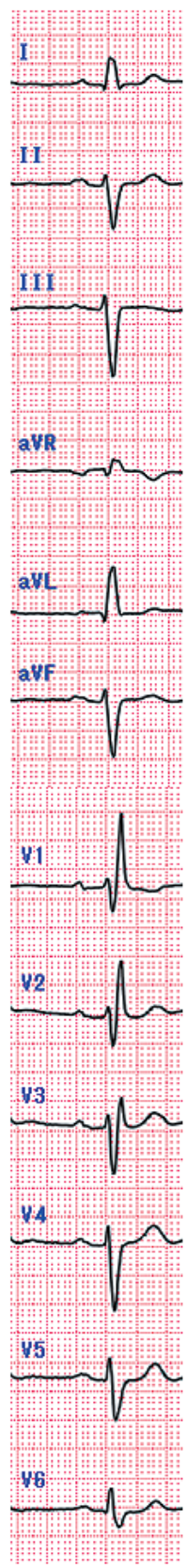

\section{E CLBBB}

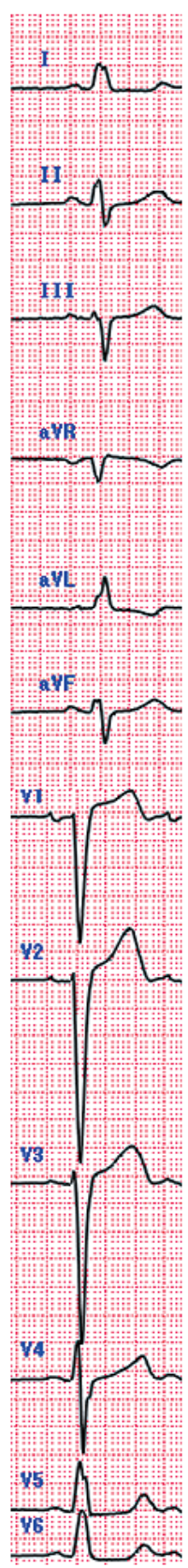

Figure 3. Classification in 12-lead electrocardiography at baseline. We divided the enrolled patients into 5 groups according to their baseline QRS morphology, i.e., normal (A), LAFB (B), CRBBB (C), CRBBB with LAFB (D), and CLBBB (E) pattern. CRBBB indicates complete right bundle branch block; CLBBB, complete left bundle branch block; and LAFB, left anterior fascicular block.

fined as left-axis deviation of $\leq-45^{\circ}$ and the presence of $\mathrm{qR}$ complex in lead I and rS complex in leads II and III. CRBBB was defined as QRS duration of $\geq 120 \mathrm{~ms}$, a secondary $\mathrm{R}$ wave greater than the initial $\mathrm{R}$ wave in the right precordial leads, and wide $\mathrm{S}$ wave in leads $\mathrm{I}$ and V6. CLBBB was defined as QRS duration of $\geq 120 \mathrm{~ms}$, the presence of monophasic QS or rS complex in lead V1, and a monophasic $\mathrm{R}$ wave in lead V6. ${ }^{17)}$
Transthoracic echocardiography: We evaluated LV wall motion abnormality by echocardiography before and after pacemaker implantation. The first assessment was performed by echocardiography at the nearest time point to the pacemaker implantation. The last assessment was performed by echocardiography at approximately 12 months after the implantation because changes in LV function were supposed to occur $12-18$ months after pacing. ${ }^{7)}$ The 


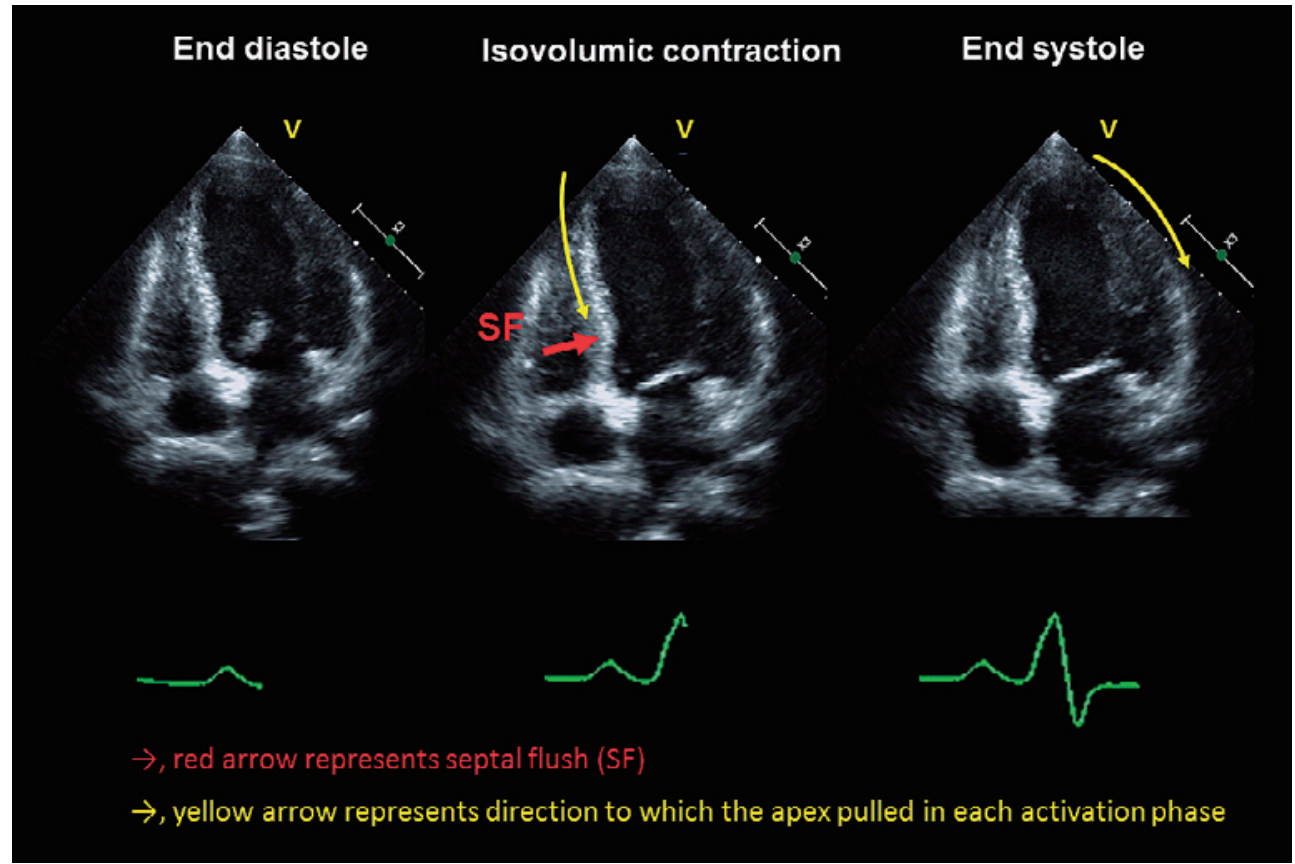

Figure 4. Schematic image representing left ventricular mechanical asynchrony. The figure shows the typical sequence of mechanical (top panels) and electrical (bottom panel) events in left ventricular asynchrony. An early electrical activation of the septum results in a short initial septal contraction and septal inward motion during the isovolumetric contraction period (within the QRS width), which is described as "septal flush." The early activation of the septum causes the apex to move septally early in systole, and the delayed activation of the lateral wall causes a subsequent lateral contraction during the ejection phase while stretching the septum (yellow arrow in the left panel). The motion to the lateral side pulls the apex laterally, resulting in septal-to-lateral apical motion, which is described as "apical rocking". CLBBB indicates complete left bundle branch block.

echocardiography was evaluated by skilled laboratory technicians who were engaged in echocardiography for at least 5 years. They were blinded to the clinical data of the patients. Transthoracic echocardiography was evaluated using a commercially available ultrasonographic system equipped with a 2- to 3-MHz imaging probe, e.g., Vivid 7 (GE Healthcare, Horten, Norway), Aplio 500 (Toshiba Medical Systems, Tokyo, Japan), and ALOKA prosound $\alpha$ 10 system (Aloka Co. Ltd, Tokyo, Japan). When the regional assessment of wall motion appeared to be in a gray zone, a technician re-evaluated the digitally stored data using commercially available software, e.g., EchoPAC PC (GE Healthcare, Horten, Norway), Toshiba systems (2D wall motion tacking, Toshiba Medical Systems, Tokyo, Japan), and Aloka Line Data format (Hitachi-Aloka, Tokyo, Japan). When the assessment differed between the examiners, they reached a consensus through discussion. Skilled doctors who had $>15$ years of experience as cardiologists controlled the examination.

Chamber quantification and wall motion evaluation were routinely performed in accordance with the American Society of Echocardiography guidelines. ${ }^{13)}$ Linear internal measurements of the LV and its walls were performed in the parasternal long-axis view using a twodimensional (2D) echocardiography-guided M-mode approach. LVDd and LV end-systolic diameter (LVDs) were measured, and LVEF was calculated by the Teicholz formula. LV wall motion was evaluated using a 17-segment model. Each segment was assessed in multiple views and a 4-grade scoring was applied: 1) normal or hyperkinetic, 2) hypokinetic, 3) akinetic, and 4) dyskinetic. ${ }^{13)}$ Local wall motion abnormality was defined as asynergy.

We visually assessed heterogeneous intra-LV contraction within the LV. LV mechanical asynchrony was defined as discoordinate contraction between opposing regions of the LV wall. Paradoxical intraventricular wall motion that bulges into the LV in early systole and is pushed into the LV in diastole was typical abnormality in contraction patterns, which was well described as apical rocking and septal flush ${ }^{18-20)}$ (Figure 4). Given that the quantitative assessment of asynchrony by tissue Doppler imaging or strain is usually performed in patients who are candidates for cardiac resynchronization therapy, we did not routinely perform those examinations in patients with normal cardiac function. Visual assessment is an important reference for decision making ${ }^{13)}$ and a potentially superior method in clinical practice. . $^{18-20)}$

Clinical outcome: We excluded 9 patients who had asynchrony at baseline, and we compared the event-free rate between patients with and without new occurrence of asynchrony under non-RV pacing. The event was defined as cardiovascular death, hospitalization due to heart failure, new-onset atrial fibrillation (AF), and device-detected AF. New-onset AF was defined as clinically apparent AF identified by 12-lead electrocardiography or 24-hour Holter monitoring. Device-detected AF was defined as any 
AF identified by a pacemaker and confirmed by an electrophysiologist. The pacemaker protocol of AF detection was programmed as atrial rate $>175 \mathrm{bpm}$ and lasting $\geq$ 20 seconds. $^{21)}$ Electrophysiologists evaluated all high-rate episodes ( $>175 \mathrm{bpm}$ ) lasting $\geq 20$ seconds documented by intracardiac electrography and judged if it was really AF. The atrial sensing configuration was bipolar, the sensitivity was basically $0.5 \mathrm{mV}$, and the post-ventricular atrial blanking period was $\geq 100 \mathrm{~ms}$ to avoid over sensing of the far-field $\mathrm{R}$ wave signals.

Statistical analysis: Normally distributed continuous variables are presented as mean \pm standard deviation and those with a non-normal distribution as median and interquartile range (IQR). Categorical variables are represented as frequency and percentage. Parametric data were compared using an unpaired or paired Student's $t$-test and non-parametric data using a Mann-Whitney $U$ test, Wilcoxon signed-rank test, chi-squared test, or Fisher's exact test, as appropriate. Univariate and multivariate logistic regression analyses were performed to assess the ECG findings associated with asynchrony motion. The incidence of clinical outcomes was compared between the patients who developed new asynchrony and those who did not by the Kaplan-Meier method and log-rank test. Multivariable Cox proportional hazards regression analysis was performed to determine the factors associated with the clinical outcomes, including new occurrence of asynchrony, age, sex, coronary artery disease, vulvular heart disease, $\mathrm{AF}$, hypertension, diabetes mellitus, chronic renal disease, beta-blocker use, and angiotensin converting enzyme inhibitors or angiotensin receptor blocker use as independent variables. Proportionality assumptions were tested by graphic approaches. Analyses were performed using MedCalc software (version 17). A $P$ value $<0.05$ was considered statistically significant.

\section{Results}

Analysis of the relationship between ECG findings and asynchrony motion:

Study patients For the analysis of the relationship between ECG findings and asynchrony motion both at baseline and under non-RVA pacing, 97 patients were included. Mean age was $76 \pm 9$ years, and $53(55 \%)$ patients were male. Given the strict criteria, the baseline cardiac function was completely normal (LVDd $=50 \pm 5 \mathrm{~mm}$ and $\mathrm{LVEF}=$ $71 \% \pm 8 \%$ ), and the cumulative percent of ventricular pacing was very high (median 98\%). The type of QRS at baseline was heterogeneous. At baseline, 70 (72\%) patients had persistent AV block, whereas 27 (28\%) patients had transient AV block. Twenty-two (23\%) patients exhibited heart failure on admission, but this was resolved by pacing treatment. The estimated RV lead position was at high-septum in 8 patients, at mid-septum in 65 patients, and at low-septum in 24 patients.

Electrocardiography and echocardiography at baseline and under non-RVA pacing Electrocardiography and echocardiography at baseline were performed at 2 days (median, IQR: 1-2) and 7 days (median, IQR: 3-16) before pacemaker implantation. Mechanical asynchrony was detected in $9(9 \%)$ patients. CLBBB was significantly asso- ciated with asynchrony both by univariate and multivariate analyses (Table I).

Electrocardiography and echocardiography under non-RVA pacing were performed on the same day at 398 days (median, IQR: 301-506) after pacemaker implantation. Electrocardiography revealed that the paced QRS duration was longer than the intrinsic QRS duration $(120 \pm$ $25 \mathrm{~ms}$ versus $154 \pm 15 \mathrm{~ms}, P<0.001)$. The majority of QRS morphology under non-RVA pacing was CLBBB pattern (Figure 2B), and the transitional zone was within V4-5. Echocardiography revealed that left atrial diameter (from $43 \pm 6 \mathrm{~mm}$ to $42 \pm 7 \mathrm{~mm}, P=0.039$ ) and LVDd (from $50 \pm 5 \mathrm{~mm}$ to $49 \pm 5 \mathrm{~mm}, P=0.033$ ) decreased under non-RVA pacing compared with those at baseline. LVDs (from $30 \pm 5 \mathrm{~mm}$ to $31 \pm 6 \mathrm{~mm}, P=0.099$ ) increased and LVEF (from $70 \% \pm 8 \%$ to $65 \% \pm 10 \%, P<$ 0.001) decreased under non-RVA pacing compared with those at baseline (Supplemental Text and Tables I-V).

The frequency of detecting LV mechanical asynchrony increased significantly $(P<0.001)$ from $9 \%$ at baseline to $39 \%$ under non-RVA pacing. Significant enlargement of LVDs was observed in patients with pacinginduced asynchrony (from $30 \pm 4 \mathrm{~mm}$ to $33 \pm 6 \mathrm{~mm}, P=$ 0.005 ) but not in those without. A significant improvement of mitral regurgitation was observed in patients without asynchrony motion but not in those with asynchrony motion (Supplemental Text and Tables I-V). By univariate analysis, LAFB, CRBBB with LAFB, and CLBBB at baseline were detected as predictors of asynchrony motion under non-RVA pacing. By multivariate analysis, LAFB and CLBBB at baseline were the predictors of asynchrony motion under non-RVA pacing (Table I).

Comparison of the ratio detecting asynchrony between patients with and without CRBBB, LAFB, or CLBBB The frequency of detecting asynchrony was not different between patients with and without CRBBB both at baseline and under non-RVA pacing (Figure 5, left panel). The frequency of detecting asynchrony was higher in patients with CLBBB than in those without CLBBB both at baseline [odds ratio $(\mathrm{OR})=20.8,95 \%$ confidence interval $(\mathrm{CI})$ $=4.21-102]$ and under non-RVA pacing $(\mathrm{OR}=7.60,95 \%$ $\mathrm{CI}=1.52-38.1)$ (Figure 5, middle panel). The frequency of detecting asynchrony was not different between patients with and without LAFB at baseline; however, it was higher in patients with LAFB than in those without under non-RVA pacing $(\mathrm{OR}=7.14,95 \% \mathrm{CI}=2.46-20.8)$ (Figure 5, right panel).

Predictors of developing pacing-induced mechanical asynchrony All patients with mechanical asynchrony at baseline had asynchrony motion under non-RVA pacing. Among the 88 patients who did not have asynchrony at baseline, LAFB at baseline was a significant predictor of new occurrence of asynchrony motion under non-RVA pacing by both univariate and multivariate analyses, whereas intrinsic QRS or paced QRS duration was not (Table I).

Analysis of the long-term clinical outcomes Excluding 9 patients who had asynchrony at baseline, the remaining 88 patients were divided into 2 groups according to the presence or absence of new asynchrony induced by non-RAV 
Table I. Analysis of ECG Findings Associated with Asynchrony Motion

\begin{tabular}{|c|c|c|c|c|c|c|}
\hline & \multirow[b]{2}{*}{ Negative } & \multirow[b]{2}{*}{ Positive } & \multicolumn{2}{|c|}{ Univariate analysis } & \multicolumn{2}{|c|}{ Multivariate analysis } \\
\hline & & & $\begin{array}{l}\text { Odds ratio } \\
{[95 \% \mathrm{CI}]}\end{array}$ & $P$ value & $\begin{array}{c}\text { Odds ratio } \\
{[95 \% \mathrm{CI}]}\end{array}$ & $P$ value \\
\hline Asynchrony at baseline, $n=97$ & $n=88(91 \%)$ & $n=9(9 \%)$ & & & & \\
\hline \multicolumn{7}{|l|}{ Baseline electrocardiographic parameters } \\
\hline 1st or 2 nd degree AV block, $n(\%)$ & $6(7)$ & $1(11)$ & $1.71[0.18-16.0]$ & 0.64 & & \\
\hline Intrinsic QRS duration, ms & $119 \pm 23$ & $134 \pm 35$ & $1.00[1.00-1.06]$ & 0.091 & & \\
\hline Narrow QRS duration ( $<120 \mathrm{~ms}), n(\%)$ & $37(42)$ & $2(22)$ & $0.39[0.08-2.00]$ & 0.26 & & \\
\hline Prolonged QRS duration ( $\geq 120 \mathrm{~ms}), n(\%)$ & $51(58)$ & $7(78)$ & $2.54[0.50-12.9]$ & 0.26 & & \\
\hline Any bundle branch block, $n(\%)$ & $53(60)$ & $7(78)$ & $2.31[0.45-11.8]$ & 0.31 & & \\
\hline Presence of LAFB, $n(\%)$ & $21(24)$ & $1(11)$ & $0.40[0.05-3.38]$ & 0.40 & & \\
\hline Presence of CRBBB, $n(\%)$ & $45(51)$ & $2(22)$ & $0.27[0.05-1.39]$ & 0.12 & & \\
\hline Presence of CRBBB with LAFB, $n(\%)$ & $18(20)$ & $1(11)$ & $0.49[0.06-4.14]$ & 0.51 & & \\
\hline Presence of CLBBB, $n(\%)$ & $5(6)$ & $5(56)$ & $20.8[4.21-102]$ & $<0.001$ & $20.8[4.21-102]$ & $<0.001$ \\
\hline Asynchrony under RVSP, $n=97$ & $n=59(61 \%)$ & $n=38(39 \%)$ & & & & \\
\hline \multicolumn{7}{|l|}{ Baseline electrocardiographic parameters } \\
\hline 1st or 2nd degree AV block, $n(\%)$ & $3(5)$ & $4(11)$ & $2.20[0.46-10.4]$ & 0.32 & & \\
\hline Intrinsic QRS duration, ms & $117 \pm 23$ & $126 \pm 27$ & $1.01[1.00-1.03]$ & 0.10 & & \\
\hline Narrow QRS duration ( $<120 \mathrm{~ms}), n(\%)$ & $26(44)$ & $13(34)$ & $0.66[0.28-1.54]$ & 0.33 & & \\
\hline Prolonged QRS duration ( $\geq 120 \mathrm{~ms}), n(\%)$ & $33(56)$ & $25(66)$ & $1.52[0.65-3.53]$ & 0.33 & & \\
\hline Any bundle branch block, $n(\%)$ & $33(56)$ & $27(71)$ & $1.93[0.81-4.61]$ & 0.14 & & \\
\hline Presence of LAFB, $n(\%)$ & $7(12)$ & $15(40)$ & $4.84[1.74-13.5]$ & 0.003 & $7.14[2.46-20.8]$ & $<0.001$ \\
\hline Presence of CRBBB, $n(\%)$ & $30(51)$ & $17(45)$ & $0.78[0.35-1.77]$ & 0.56 & & \\
\hline Presence of CRBBB with LAFB, $n(\%)$ & $7(12)$ & $12(32)$ & $3.43[1.21-9.74]$ & 0.021 & & \\
\hline Presence of CLBBB, $n(\%)$ & $2(3)$ & $8(21)$ & $7.60[1.52-38.1]$ & 0.014 & $13.3[2.55-69.7]$ & 0.002 \\
\hline \multicolumn{7}{|l|}{ Electrocardiographic parameters under RVSP } \\
\hline Paced QRS duration, ms & $152 \pm 16$ & $156 \pm 11$ & $1.02[0.99-1.05]$ & 0.20 & & \\
\hline Transitional zone score & $4.5 \pm 1.0$ & $4.7 \pm 0.7$ & $1.29[0.80-2.10]$ & 0.29 & & \\
\hline Amplitude of QRS wave in lead I, mV & $0.4 \pm 0.3$ & $0.5 \pm 0.4$ & $2.55[0.74-8.81]$ & 0.14 & & \\
\hline Amplitude of QRS wave in lead II, mV & $0.6 \pm 0.8$ & $0.5 \pm 0.5$ & $0.74[0.40-1.37]$ & 0.34 & & \\
\hline New occurrence of asynchrony, $n=88$ & $n=57(65 \%)$ & $n=31(35 \%)$ & & & & \\
\hline \multicolumn{7}{|l|}{ Baseline electrocardiographic parameters } \\
\hline $1^{\text {st }}$ or $2^{\text {nd }}$ degree AV block, $n(\%)$ & $4(6)$ & $3(10)$ & $1.93[0.37-10.2]$ & 0.44 & & \\
\hline Intrinsic QRS duration, ms & $118 \pm 22$ & $121 \pm 25$ & $1.01[0.99-1.03]$ & 0.53 & & \\
\hline Narrow QRS duration $(<120 \mathrm{~ms}), n(\%)$ & $25(44)$ & $12(39)$ & $0.81[0.33-1.97]$ & 0.64 & & \\
\hline Prolonged QRS duration ( $\geq 120 \mathrm{~ms}), n(\%)$ & $32(56)$ & $19(61)$ & $1.24[0.51-3.01]$ & 0.64 & & \\
\hline Any bundle branch block, $n(\%)$ & $39(59)$ & $22(68)$ & $1.78[0.70-4.54]$ & 0.23 & & \\
\hline Presence of LAFB, $n(\%)$ & $7(12)$ & $14(45)$ & $5.88[2.04-17.0]$ & 0.001 & $5.88[2.04-17.0]$ & 0.001 \\
\hline Presence of CRBBB, $n(\%)$ & $30(51)$ & $16(5)$ & $1.03[0.43-2.47]$ & 0.95 & & \\
\hline Presence of CRBBB with LAFB, $n(\%)$ & $7(12)$ & $12(39)$ & $4.51[1.54-13.2]$ & 0.006 & & \\
\hline Presence of CLBBB, $n(\%)$ & $2(4)$ & $3(10)$ & $2.95[0.47-18.7]$ & 0.25 & & \\
\hline \multicolumn{7}{|l|}{ Electrocardiographic parameters under RVSP } \\
\hline Paced QRS duration, ms & $153 \pm 16$ & $156 \pm 12$ & $1.02[0.98-1.05]$ & 0.33 & & \\
\hline Transitional zone score & $4.5 \pm 1.0$ & $4.7 \pm 0.7$ & $1.30[0.78-2.16]$ & 0.31 & & \\
\hline Amplitude of QRS wave in lead I, mV & $0.4 \pm 0.3$ & $0.5 \pm 0.4$ & $1.63[0.43-6.12]$ & 0.47 & & \\
\hline Amplitude of QRS wave in lead II, mV & $0.6 \pm 0.8$ & $0.5 \pm 0.6$ & $0.79[0.42-1.50]$ & 0.48 & & \\
\hline
\end{tabular}

We compared various parameters on 12-lead electrocardiogram at baseline and under RVSP. Multivariate stepwise logistic regression analysis was performed to assess the factors that had an influence on asynchrony with entry and stay criteria of 0.05 and 0.10 . We also evaluated the parameters depending on new occurrence of asynchrony, excluding 9 patients who had asynchrony at baseline. AV indicates atrioventricular; CI, confidence interval; CLBBB, complete left bundle branch block; CRBBB, complete right bundle branch block; ECG, electrocardiogram; and LAFB, left anterior fascicular block.

pacing and were compared in terms of the clinical outcomes. Patients' characteristics of those 88 patients are presented in Table II.

During a median follow-up period of 4.8 years (IQR; 3.3-6.7 years), the incidence of cardiovascular death, hospitalization due to heart failure, new-onset $\mathrm{AF}$, and devicedetected $\mathrm{AF}$ was $9.1 \%, 14.8 \%, 12.4 \%$, and $37.0 \%$, respectively. There were no significant differences in the inci- dence of cardiovascular death [hazard ratio $(\mathrm{HR})=1.41$, 95\% CI $=0.31-6.41, P=0.63]$, hospitalization due to heart failure $(\mathrm{HR}=1.18,95 \% \mathrm{CI}=0.38-3.71, P=0.77)$, or new-onset $\mathrm{AF}(\mathrm{HR}=1.79,95 \% \mathrm{CI}=0.49-6.50, P=$ 0.35 ) between patients with and without new occurrence of asynchrony under non-RVA pacing. However, the incidence of device-detected AF was significantly more frequent in patients who developed pacing-induced asyn- 

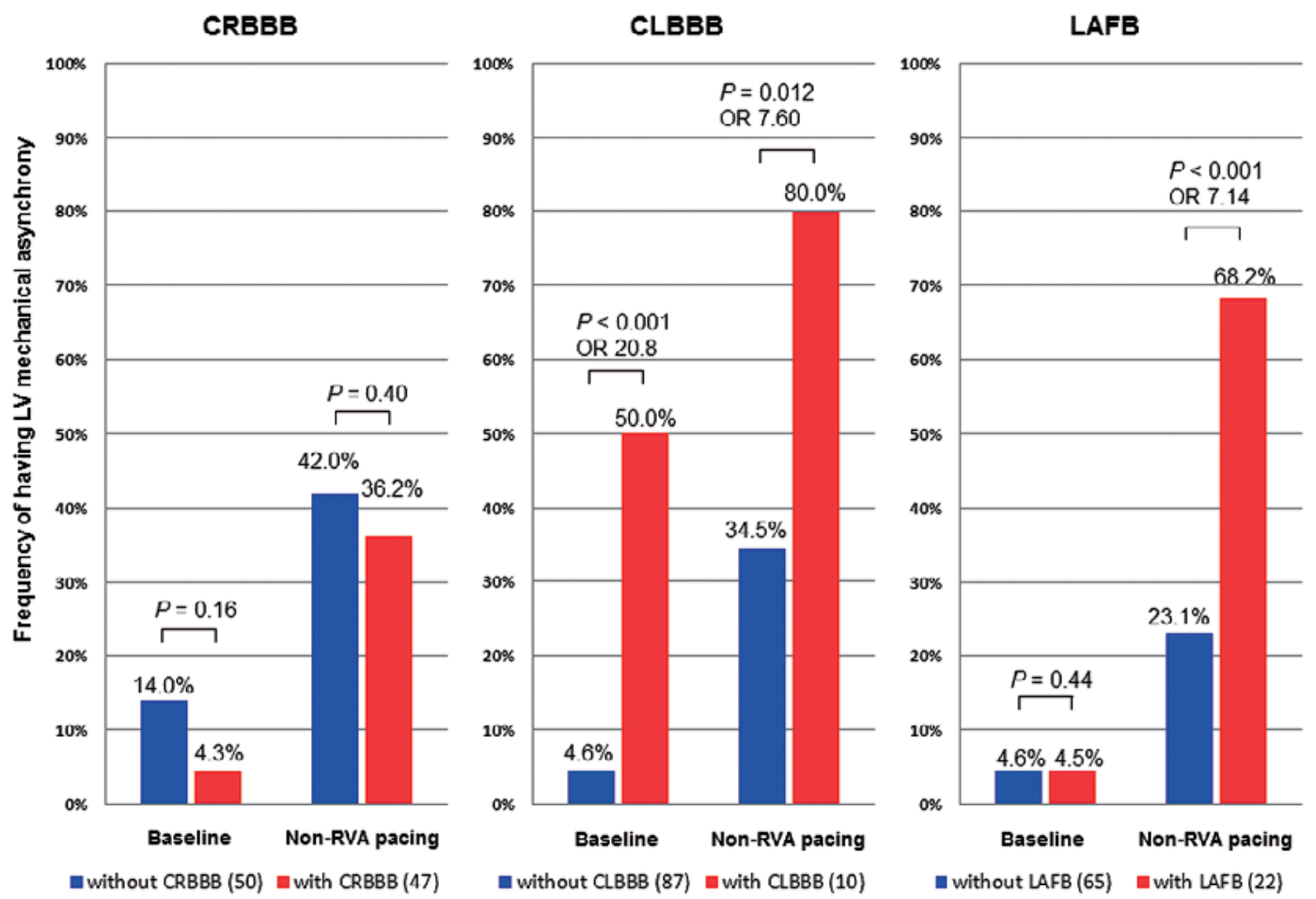

Figure 5. Comparison of the frequency for detecting asynchrony motion. The frequency of detecting LV mechanical asynchrony was compared at baseline and under non-RVA pacing by the presence or absence of specific bundle branch or fascicular conduction abnormality. CI indicates confidence interval; CRBBB, complete right bundle branch block; CLBBB, complete left bundle branch block; LAFB, left anterior fascicular block; LV, left ventricular; non-RVA pacing, right ventricular non-apical pacing; and $\mathrm{OR}$, odds ratio.

chrony than in those who did not $(53.3 \%$ versus $27.5 \%$, $\mathrm{HR}=2.17,95 \% \mathrm{CI}=1.02-4.61, P=0.03$ ) (Figure 6). Multivariate Cox regression analysis also revealed that pacing-induced asynchrony is a significant predictor of device-detected AF (Table III).

\section{Discussion}

RVA pacing is known to cause an inhomogeneous contraction of the LV myocardium with abnormal apical septal contractility, resulting in heart failure in some patients. ${ }^{22)}$ The mechanical asynchrony induced by RVA pacing was found to be enhanced by the presence of baseline conduction abnormality in patients with poor LV function. ${ }^{10-12)}$ In contrast, the mechanical asynchrony might be resolved by placing the lead on the RV septum, resulting in less LV mechanical asynchrony and better longitudinal function than the results if the lead was placed on the RV apex. $^{4-9)}$ However, even in patients with normal cardiac function and under non-RVA pacing, a fraction exhibits asynchrony motion. The clinical importance and predictors of asynchrony in such a "low-risk" population were not well described previously because of the estimated good prognosis in clinical practice.

In the present study, we examined the predictive factors and clinical significance of asynchrony motion in patients with normal cardiac function. Asynchrony motion was observed in only $9 \%$ of patients at baseline but in $39 \%$ of patients under non-RVA pacing. CLBBB was found to be significantly associated with asynchrony at baseline; LAFB at baseline was found to be a significant predictor of developing pacing-induced asynchrony. The incidence of device-detected AF was significantly higher in patients developing pacing-induced asynchrony than in those not developing this, during a median follow-up period of 4.8 years.

The present study was considerably different from previous reports in 3 ways. First, we examined patients with entirely normal cardiac function. Second, we examined pacing-induced asynchrony under non-RVA pacing (estimated septum pacing). Third, we examined the influence of hemiblock (LAFB and left posterior fascicular block). Finally, we showed a contribution of LAFB to the new occurrence of pacing-induced asynchrony.

Lack of correlation between paced QRS complex and asynchrony motion: In the present study, although LAFB at baseline was associated with the occurrence of nonRVA pacing-induced asynchrony, no single electrocardiography criterion under non-RVA pacing (including paced QRS duration, transitional zone, and amplitude in leads I and II) could accurately differentiate patients with and without asynchrony motion. ${ }^{12)}$

In electrocardiographic analysis, paced QRS duration, presence of precordial axis transition, notching, and leftaxis deviation were useful parameters to predict asynchrony under RVA pacing. ${ }^{12)}$ However, the correlation between each variable and asynchrony was weak, and its clinical utility to predict asynchrony was limited. An 
Table II. Baseline Characteristics of Study Patients

\begin{tabular}{|c|c|c|c|c|}
\hline & $\begin{array}{l}\text { Total } \\
n=88\end{array}$ & $\begin{array}{c}\text { Patients with } \\
\text { new asynchrony } \\
n=31\end{array}$ & $\begin{array}{c}\text { Patients without } \\
\text { new asynchrony } \\
n=57\end{array}$ & $P$ value \\
\hline Age, years & $76 \pm 10$ & $77 \pm 7$ & $76 \pm 11$ & 0.65 \\
\hline Male, $n(\%)$ & $48(55)$ & $19(61)$ & $29(51)$ & 0.35 \\
\hline Body mass index, $\mathrm{kg} / \mathrm{m}^{2}$ & $22 \pm 4$ & $22 \pm 3$ & $22 \pm 4$ & 0.66 \\
\hline eGFR, $\mathrm{mL} /$ minute $/ 1.73 \mathrm{~m}^{2}$ & $56 \pm 19$ & $56 \pm 22$ & $56 \pm 18$ & 0.98 \\
\hline \multicolumn{5}{|l|}{ Baseline diseases, $n(\%)$} \\
\hline Heart failure on admission & $20(23)$ & $6(19)$ & $14(25)$ & 0.58 \\
\hline Coronary artery disease* & $30(34)$ & $8(26)$ & $22(39)$ & 0.23 \\
\hline Valvular heart disease $^{\dagger}$ & $10(11)$ & $3(16)$ & $7(12)$ & 0.71 \\
\hline Prior myocardial infarction & $9(9)$ & $1(3)$ & $8(14)$ & 0.15 \\
\hline Atrial fibrillation & $7(7)$ & $1(3)$ & $6(11)$ & 0.41 \\
\hline Hypertension & $65(67)$ & $19(61)$ & $40(70)$ & 0.40 \\
\hline Diabetes mellitus & $11(13)$ & $4(13)$ & $7(12)$ & 0.93 \\
\hline Chronic kidney disease & $15(17)$ & $7(23)$ & $8(14)$ & 0.31 \\
\hline \multicolumn{5}{|l|}{ Medication at discharge, $n(\%)$} \\
\hline Calcium channel blocker & $37(42)$ & $11(36)$ & $26(46)$ & 0.36 \\
\hline ACE-inhibitor/ARB & $40(46)$ & $13(42)$ & $27(47)$ & 0.63 \\
\hline Beta-blocker & $16(18)$ & $3(10)$ & $13(23)$ & 0.13 \\
\hline Diuretics & $11(13)$ & $3(10)$ & $8(14)$ & 0.74 \\
\hline Class I AAD & $6(7)$ & $1(3)$ & $5(9)$ & 0.42 \\
\hline Class III AAD & $3(3)$ & $1(3)$ & $2(4)$ & 1.00 \\
\hline Antiplatelet & $25(28)$ & $7(23)$ & $18(32)$ & 0.37 \\
\hline Anticoagulant & $10(11)$ & $1(3)$ & $9(16)$ & 0.09 \\
\hline \multicolumn{5}{|l|}{ Echocardiographic parameters } \\
\hline Left atrial diameter, $\mathrm{mm}$ & $43 \pm 6$ & $42 \pm 5$ & $44 \pm 6$ & 0.30 \\
\hline Left ventricular end-diastolic diameter, $\mathrm{mm}$ & $50 \pm 4$ & $51 \pm 4$ & $50 \pm 4$ & 0.11 \\
\hline Left ventricular end-systolic diameter, mm & $30 \pm 5$ & $30 \pm 4$ & $29 \pm 5$ & 0.48 \\
\hline Left ventricular ejection fraction, $\%$ & $71 \pm 8$ & $71 \pm 6$ & $71 \pm 8$ & 0.82 \\
\hline Mitral regurgitation, $n(\%)$ (Seller's classification grade $\geq 2$ ) & $12(14)$ & $5(16)$ & $7(12)$ & 0.62 \\
\hline Asynergy, $n(\%)$ & $9(9)$ & $1(3)$ & $8(14)$ & 0.15 \\
\hline \multicolumn{5}{|l|}{ Pacing rate, $\%$} \\
\hline Cum\% Ap & $26 \pm 25$ & $23 \pm 20$ & $28 \pm 28$ & 0.32 \\
\hline Cum\% Vp & $98 \pm 5$ & $99 \pm 2$ & $98 \pm 6$ & 0.13 \\
\hline \multicolumn{5}{|l|}{ Estimated RV lead position, $n(\%)$} \\
\hline High-septum & $7(8)$ & $1(3)$ & $6(11)$ & 0.43 \\
\hline Mid-septum & $57(65)$ & $22(71)$ & $35(61)$ & \\
\hline Low-septum & $24(27)$ & $8(26)$ & $16(28)$ & \\
\hline \multicolumn{5}{|l|}{ Electrocardiographic parameters under pacing } \\
\hline Paced QRS duration, ms & $154 \pm 15$ & $156 \pm 12$ & $153 \pm 16$ & 0.33 \\
\hline Transitional zone score & $4.5 \pm 0.9$ & $4.7 \pm 0.7$ & $4.5 \pm 1.0$ & 0.31 \\
\hline Amplitude of QRS wave in lead I, mV & $0.5 \pm 0.3$ & $0.5 \pm 0.4$ & $0.4 \pm 0.3$ & 0.47 \\
\hline Amplitude of QRS wave in lead II, $\mathrm{mV}$ & $0.6 \pm 0.7$ & $0.5 \pm 0.6$ & $0.6 \pm 0.8$ & 0.48 \\
\hline
\end{tabular}

AAD indicates anti-arrhythmic drug; ACE, angiotensin-converting enzyme; ARB, angiotensin II receptor blocker; AV, atrioventricular; CLBBB, complete left bundle branch block; CRBBB, complete right bundle branch block; Cum\% Ap, cumulative percent atrial pacing; Cum\% Vp, cumulative percent ventricular pacing; eGFR, estimated glomerular filtration rate; HbAlc, hemoglobin A1c; LAFB, left anterior fascicular block; and RV, right ventricular. *Coronary artery disease means the presence of stenosis $>50 \%$ diagnosed by MDCT and stenosis $>75 \%$ diagnosed by coronary angiography, and/or history of any coronary angioplasty. ${ }^{\dagger}$ Patients with valvular heart disease are defined as those who suffer from irreversible moderate to severe valve insufficiency and those who received valve surgery. One patient received aortic valve replacement, 2 patients received mitral valve repair, and the other 7 patients suffered from mitral regurgitation.

analysis of patients with heart failure demonstrated intraventricular asynchrony in two-thirds of patients with wide QRS and in one-third of patients with narrow $\mathrm{QRS}{ }^{23)} \mathrm{A}$ relaxation delay, but not an activation delay, was an advocated possible mechanism to explain asynchrony in patients with narrow QRS duration. ${ }^{2-6,24)}$ It was difficult to judge the existence of relaxation abnormality from a 12lead ECG.

Influence of LAFB on asynchrony: The present study demonstrates that intrinsic LAFB is not associated with asynchrony motion at baseline but with asynchrony motion under non-RVA pacing. The different electromechanical delays under intrinsic rhythm and non-RVA pacing would be caused by different action potential propagations within the LV.

According to an examination of endocardial RV and LV activation sequences using a 3D electroanatomic contact mapping system, LAFB on surface electrocardiogra- 

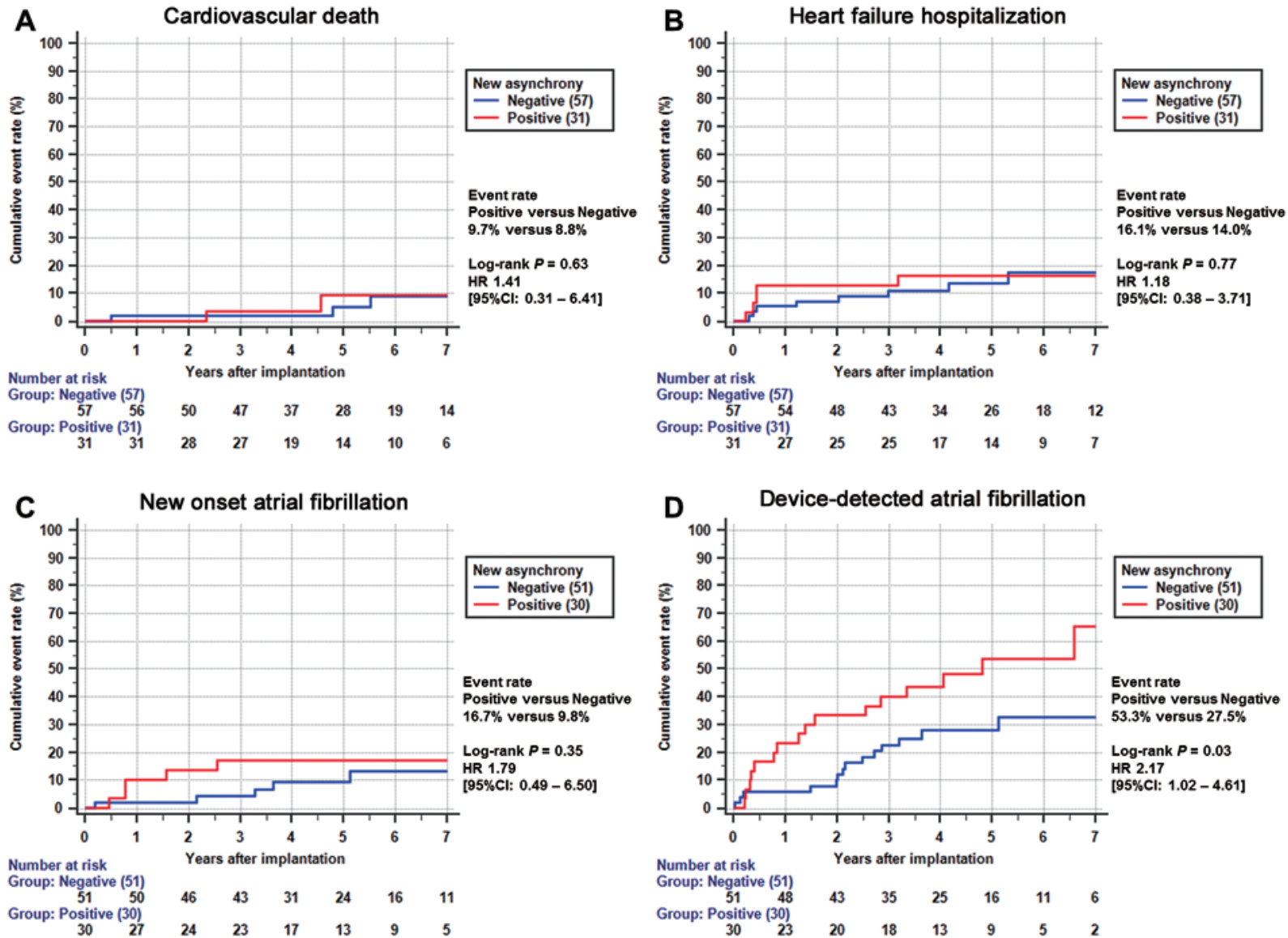

Figure 6. Kaplan-Meier survival curve analysis evaluating incidence of cardiovascular events. The incidence of cardiovascular death (A), hospitalization due to heart failure (B), new-onset $\mathrm{AF}(\mathbf{C})$, and device-detected $\mathrm{AF}(\mathbf{D})$ was compared between patients developing pacing-induced asynchrony and their counterparts. Of 88 patients, 7 had a history of atrial fibrillation and were excluded from the evaluation of new-onset AF and device-detected AF. AF indicates atrial fibrillation; CI, confidence interval; and HR, hazard ratio.

Table III. Multivariate Cox Regression Analysis

\begin{tabular}{lcc}
\hline & HR $(95 \% \mathrm{CI})$ & $P$ value \\
\hline A. Cardiovascular death & $1.25[1.05-1.49]$ & 0.011 \\
$\quad$ Age & $7.30[1.24-42.9]$ & 0.028 \\
$\quad$ Male gender & & \\
& $4.84[1.48-15.9]$ & 0.009 \\
$\begin{array}{l}\text { B. Heart failure hospitalization } \\
\quad \text { Valvular heart disease }\end{array}$ & \\
$\begin{array}{l}\text { C. New onset atrial fibrillation } \\
\text {-* }\end{array}$ & - \\
$\begin{array}{l}\text { D. Device-detected atrial fibrillation } \\
\quad \text { New occurrence of asynchrony }\end{array}$ & $2.14[1.04-4.40]$ & 0.048 \\
$\quad$ Valvular heart disease & $3.00[1.13-7.98]$ & 0.033 \\
\hline
\end{tabular}

The analysis was performed to examine factors associated with each clinical outcome. New occurrence of asynchrony, age, sex, coronary artery disease, valvular heart disease, hypertension, diabetes mellitus, beta-blocker use, ACE-inhibitor, and/or ARB use were included as variables. New onset atrial fibrillation and device-detected atrial fibrillation were examined in 81 patients excluding 7 patients who already had documented atrial fibrillation. *We could not identify significant predictors of new onset atrial fibrillation. ACE indicates angiotensin-converting enzyme; ARB, angiotensin II receptor blocker; CI, confidence interval; and HR, hazard ratio. phy was correlated with a single LV breakthrough site at the inferior septum, whereas $2 \mathrm{LV}$ breakthrough sites (the inferior septum and basal anterior wall) were observed in patients without LAFB pattern. ${ }^{25)}$ This difference may result from heterogeneous conduction velocity between the impaired left anterior fascicle and unimpaired posterior fascicle. However, in a previous study that enrolled candidates for cardiac resynchronization therapy (CRT) with CRBBB, intra-LV electromechanical delay before CRT was not different between patients with and without LAFB ${ }^{26}$ ) which was consistent with the findings of the present study that included patients with normal cardiac function.

In contrast, the present study reveals that intrinsic LAFB is associated with developing asynchrony motion under non-RVA pacing. RV pacing in patients with heart failure has been reported to increase the electrical activation delays within the LV that were enhanced by accompanying conduction disease. ${ }^{12)}$ In the post hoc analysis of the Multicenter Automatic Defibrillator Implantation Trial with CRT, patients with RBBB and LAFB had worse response to CRT with less improvement in LV volumes and function than those without LAFB. ${ }^{27)}$ The greater activation delays by RV pacing in patients with both RBBB and 
LAFB than in those with RBBB alone might have enhanced the intra-LV mechanical asynchrony, resulting in a worsened response to CRT.

Clinical implication of LAFB:

Atrial fibrillation In the present study, the incidence of device-detected AF was significantly higher in the patients who developed asynchrony under non-RVA pacing than in those who did not. Mitral regurgitation ${ }^{28)}$ and LV diastolic dysfunction $^{29)}$ are considered major mechanisms that explain how LV asynchrony motion causes AF. The significant improvement of mitral regurgitation detected in patients without asynchrony under non-RVA pacing, possibly due to the recovery from heart failure, was prevented in patients with asynchrony, which might be a mechanism of how asynchrony increases AF (Supplemental Text and Tables I-V).

The Asymptomatic AF and Stroke Evaluation in Pacemaker Patients and the AF Reduction Atrial Pacing Trial (ASSERT) examined the clinical importance of subclinical device-detected AF, which demonstrated that $15.7 \%$ of patients with device-detected non-symptomatic AF developed clinically apparent AF during the mean follow-up period of 2.5 years. ${ }^{30)}$ The trial also revealed that subclinical device-detected AF is associated with a significantly increased risk of ischemic stroke or systemic embolism. Although conventional clinical risk presented by the CHADS2 score or CHA2DS2-VASc score is important to determine the need for anticoagulation, risk of stroke is multifactorial ${ }^{31)}$ and device-detected AF may be an additional factor for risk stratification. ${ }^{32,33)}$ Therefore, the presence of LAFB may also be an additional factor that should be included in the risk stratification of ischemic stroke in order to identify those who would have benefited from oral anticoagulation therapy.

Cardiovascular death and heart failure In the present study, new occurrence of asynchrony motion had no significant influence on cardiovascular death and hospitalization due to heart failure. The results made us recall the fact that the clinical outcome of patients with RVA pacing has been reported differently depending on the LV function. Low LVEF at baseline has been shown to be a predictor of LV mechanical asynchrony after RVA pacing, ${ }^{11,34)}$ and the incidence of hospitalization due to heart failure was reportedly higher in patients with LV asynchrony than in those without it among patients with low LVEF. ${ }^{35}$ In contrast, little is known about the influence of pacinginduced asynchrony on heart failure in patients with normal cardiac function.

The Protection of Left Ventricular Function During Right Ventricular Pacing (PROTECT-PACE) study ${ }^{6,36}$ examined cardiac function and clinical outcomes in patients with normal cardiac function who were dependent on pacemakers. Although LV asynchrony was more frequent in patients with RVA pacing than in those with non-RVA pacing, the change in LVEF, incidence of all-cause death, or hospitalization due to heart failure were not different between the groups. In the present study, the incidence of LV asynchrony under non-RVA pacing was $32 \%$, which was lower than that previously reported in patients under RVA pacing (45\%). ${ }^{10)}$ No significant impact of LV asynchrony on cardiovascular death and heart failure obtained in the present study was consistent with the results of the PROTECT-PACE study.

Limitations: A small sample size with a low frequency of events during follow-up was a limitation of the present study. The retrospective, observational method based on transthoracic echocardiography reports made the evaluation of inter- and intra-observer variability difficult.

Quantitative measurement using tissue Doppler imaging or 2D speckle-tracking strain imaging was not used in the present study to evaluate mechanical asynchrony. ${ }^{12)}$ However, an extremely high concordance has been reported between the qualitative visual evaluation of LV wall motion by experienced cardiologists and the results of automated quantitative measurement of myocardial wall deformation. ${ }^{37)}$ Furthermore, visually assessed mechanical asynchrony was reported comparable with a regional strain pattern analysis $\left.{ }^{6}\right)$ and with anteroseptal to posterior time-to-peak radial strain difference. ${ }^{2)}$

Analysis of QRS was performed in escape rhythm in $70(72 \%)$ patients with permanent $\mathrm{AV}$ block at baseline and was performed during intrinsic rhythm in the remaining 27 patients with transient AV block. Adopting QRS morphology of different timing might have influenced the results. However, we compared QRS morphology between before and after AV block in 45 patients who underwent 12-lead electrocardiography at both time points and found that they were the same in $91 \%$ of patients (Supplemental Figures 1 and 2).

Echocardiography under non-RVA pacing was performed $>1$ year after the implantation; ${ }^{8)}$ thus, we should consider the existence of confounders such as a timedependent progression of concomitant conduction disturbance or underlying heart disease. However, according to the Cardiovascular Health Study, which is a prospective cohort study of individuals aged 65 years or older without clinically manifested cardiovascular disease, no participants $(0 \%)$ with LAFB went on to have CLBBB and only $2(0.1 \%)$ exhibited pacing among the eligible 1664 participants through 10 years of annual ECGs. ${ }^{38)}$ Thus, the progression of conduction disease was rare as a natural course, and the impact of LAFB on asynchrony might presumably be due to the pacing effect. Furthermore, high incidence of $\mathrm{AF}$, heart failure, and death was reported in patients with LAFB in the Cardiovascular Health Study cohort. ${ }^{38)}$ The clinical influence of new asynchrony motion in this study might merely reflect the poor prognosis of patients with LAFB.

\section{Conclusion}

In patients with normal cardiac function, the presence of LAFB at baseline was significantly associated with new occurrence of LV asynchrony under non-RVA pacing. The asynchrony had no significant influence on cardiovascular death, hospitalization due to heart failure, or new-onset $\mathrm{AF}$ in the long-term follow-up. However, the incidence of device-detected AF was significantly higher in patients who developed asynchrony by non-RVA pacing. 


\section{Acknowledgment}

The authors are deeply grateful to Dr. Hiroki Oe (Sakurabashi Watanabe Hospital) for kind advice on echocardiographic description.

\section{Disclosures}

\section{Conflicts of interest: None.}

\section{References}

1. Lieberman R, Padeletti L, Schreuder J, et al. Ventricular pacing lead location alters systemic hemodynamics and left ventricular function in patients with and without reduced ejection fraction. J Am Coll Cardiol 2006; 48: 1634-41.

2. Tops LF, Suffoletto MS, Bleeker GB, et al. Speckle-tracking radial strain reveals left ventricular dyssynchrony in patients with permanent right ventricular pacing. J Am Coll Cardiol 2007; 50: $1180-8$

3. Tops LF, Trines SA, Marsan N, Holman ER, Schalij MJ, Bax JJ. Acute effects of right ventricular apical pacing on left ventricular synchrony and mechanics. Circ Arrhythm Electrophysiol 2009; 2: 135-45

4. Yoshikawa H, Suzuki M, Tezuka N, Otsuka T, Sugi K. Differences in left ventricular dyssynchrony between high septal pacing and apical pacing in patients with normal left ventricular systolic function. J Cardiol 2010; 56: 44-50.

5. Inoue K, Okayama H, Nishimura K, et al. Right ventricular septal pacing preserves global left ventricular longitudinal function in comparison with apical pacing: analysis of speckle tracking echocardiography. Circ J 2011; 75: 1609-15.

6. Saito M, Kaye G, Negishi K, et al. Dyssynchrony, contraction efficiency and regional function with apical and non-apical RV pacing. Heart 2015; 101: 600-8

7. Tse HF, Yu C, Wong KK, et al. Functional abnormalities in patients with permanent right ventricular pacing: the effect of sites of electrical stimulation. J Am Coll Cardiol 2002; 40: 1451-8.

8. Takemoto Y, Hasebe H, Osaka T, et al. Right ventricular septal pacing preserves long-term left ventricular function via minimizing pacing-induced left ventricular dyssynchrony in patients with normal baseline QRS duration. Circ J 2009; 73: 1829-35.

9. Leong DP, Mitchell AM, Salna I, et al. Long-term mechanical consequences of permanent right ventricular pacing: effect of pacing site. J Cardiovasc Electrophysiol 2010; 21: 1120-6.

10. Pastore G, Noventa F, Piovesana P, et al. Left ventricular dyssynchrony resulting from right ventricular apical pacing: relevance of baseline assessment. Pacing Clin Electrophysio 2008; 31: 1456-62.

11. Varma N. Left ventricular conduction delays induced by right ventricular apical pacing: effect of left ventricular dysfunction and bundle branch block. J Cardiovasc Electrophysiol 2008; 19: 114-22.

12. Schmidt $\mathrm{M}$, Rittger $\mathrm{H}$, Marschang $\mathrm{H}$, et al. Left ventricular dyssynchrony from right ventricular pacing depends on intraventricular conduction pattern in intrinsic rhythm. Eur J Echocardiogr 2009; 10: 776-83.

13. Lang RM, Badano LP, Mor-Avi V, et al. Recommendations for cardiac chamber quantification by echocardiography in adults: an update from the American Society of Echocardiography and the European Association of Cardiovascular Imaging. J Am Soc Echocardiogr 2015; 28: 1-39.e14.

14. Pang BJ, Joshi SB, Lui EH, et al. Validation of conventional fluoroscopic and ECG criteria for right ventricular pacemaker lead position using cardiac computed tomography. Pacing Clin Electrophysiol 2014; 37: 495-504

15. Hayashi Y, Takagi M, Kakihara J, et al. Impact of simple elec- trocardiographic markers as predictors for deterioration of left ventricular function in patients with frequent right ventricular apical pacing. Heart Vessels 2018; 33: 299-308.

16. Inoue $\mathrm{K}$, Okayama $\mathrm{H}$, Nishimura $\mathrm{K}$, et al. Right ventricular septal pacing preserves global left ventricular longitudinal function in comparison with apical pacing: analysis of speckle tracking echocardiography. Circ J 2011; 75: 1609-15.

17. Elizari MV, Acunzo RS, Ferreiro M. Hemiblocks revisited. Circulation 2007; 115: 1154-63.

18. Jansen AH, van Dantzig Jm, Bracke F, et al. Qualitative observation of left ventricular multiphasic septal motion and septalto-lateral apical shuffle predicts left ventricular reverse remodeling after cardiac resynchronization therapy. Am J Cardiol 2007; 99: 966-9.

19. Stankovic I, Prinz C, Ciarka A, et al. Relationship of visually assessed apical rocking and septal flash to response and longterm survival following cardiac resynchronization therapy (PREDICT-CRT). Eur Heart J Cardiovasc Imaging 2016; 17: 262-9.

20. Sakamaki F, Seo Y, Atsumi A, et al. Novel dyssynchrony evaluation by M-mode imaging in left bundle branch block and the application to predict responses for cardiac resynchronization therapy. J Cardiol 2014; 64: 199-206.

21. Purerfellner H, Gillis AM, Holbrook R, Hettrick DA. Accuracy of atrial tachyarrhythmia detection in implantable devices with arrhythmia therapies. Pacing Clin Electrophysiol 2004; 27: 98392.

22. Sweeney MO, Hellkamp AS, Ellenbogen KA, et al. Mode Selection Trial Investigators. Adverse effect of ventricular pacing on heart failure and atrial fibrillation among patients with normal baseline QRS duration in a clinical trial of pacemaker therapy for sinus node dysfunction. Circulation 2003; 107: 2932-7.

23. Lee KH, Cho JG, Park HW, et al. QRS morphology and ventricular dyssynchrony in patients with chronic right ventricular pacing. Int J Cardiol 2014; 176: 962-8.

24. Schuster I, Habib G, Jego C, et al. Diastolic asynchrony is more frequent than systolic asynchrony in dilated cardiomyopathy and is less improved by cardiac resynchronization therapy. J Am Coll Cardiol 2005; 46: 2250-7.

25. Fantoni C, Kawabata M, Massaro R, et al. Right and left ventricular activation sequence in patients with heart failure and right bundle branch block: a detailed analysis using threedimensional non-fluoroscopic electroanatomic mapping system. J Cardiovasc Electrophysiol 2005; 16: 112-9.

26. Haghjoo M, Bagherzadeh A, Farahani MM, Haghighi ZO, SadrAmeli MA. Significance of QRS morphology in determining the prevalence of mechanical dyssynchrony in heart failure patients eligible for cardiac resynchronization: particular focus on patients with right bundle branch block with and without coexistent left-sided conduction defects. Europace 2008; 10: 566-71.

27. Tompkins C, Kutyifa V, McNitt S, et al. Effect on cardiac function of cardiac resynchronization therapy in patients with right bundle branch block (from the Multicenter Automatic Defibrillator Implantation Trial With Cardiac Resynchronization Therapy [MADIT-CRT] trial). Am J Cardiol 2013; 112: 525-9.

28. Soyama A, Kono T, Mishima T, et al. Intraventricular dyssynchrony may play a role in the development of mitral regurgitation in dilated cardiomyopathy. J Card Fail 2005; 11: 631-7.

29. Rosenberg MA, Manning WJ. Diastolic dysfunction and risk of atrial fibrillation: a mechanistic appraisal. Circulation 2012; 126: $2353-62$.

30. Healey JS, Connolly SJ, Gold MR, et al. Subclinical atrial fibrillation and the risk of stroke. N Engl J Med 2012; 366: 120-9.

31. Wu JT, Wang SL, Chu YJ, et al. Usefulness of a combination of interatrial block and a high CHADS2 score to predict new onset atrial fibrillation. Int Heart J 2016; 57: 580-5.

32. Shanmugam N, Boerdlein A, Proff J, et al. Detection of atrial high-rate events by continuous home monitoring: clinical significance in the heart failure-cardiac resynchronization therapy population. Europace 2012; 14: 230-7. 
33. Boriani G, Botto GL, Padeletti L, et al. Improving stroke risk stratification using the CHADS2 and CHA2DS2-VASc risk scores in patients with paroxysmal atrial fibrillation by continuous arrhythmia burden monitoring. Stroke 2011; 42: 1768-70.

34. Ooka J, Tanaka H, Hatani Y, et al. Risk stratification of future left ventricular dysfunction for patients with indications for right ventricular pacing due to bradycardia. Int Heart J 2017; 58: 724-30.

35. Bader H, Garrigue S, Lafitte S, et al. Intra-left ventricular electromechanical asynchrony. A new independent predictor of severe cardiac events in heart failure patients. J Am Coll Cardio 2004; 43: 248-56.

36. Kaye GC, Linker NJ, Marwick TH, et al. Effect of right ventricular pacing lead site on left ventricular function in patients with high-grade atrioventricular block: results of the ProtectPace study. Eur Heart J 2015; 36: 856-62.

37. Takahashi M, Harada N, Isozaki Y, et al. Efficiency of quantita- tive longitudinal peak systolic strain values using automated function imaging on transthoracic echocardiogram for evaluating left ventricular wall motion: new diagnostic criteria and agreement with naked eye evaluation by experienced cardiologist. Int J Cardiol 2013; 167: 1625-31.

38. Mandyam MC, Soliman EZ, Heckbert SR, Vittinghoff E, Marcus GM. Long-term outcomes of left anterior fascicular block in the absence of overt cardiovascular disease. JAMA 2013; 309: 1587-8.

\section{Supplemental Files}

Supplemental Text

Supplemental Tables I-V

Supplemental Figures 1 and 2

Please see supplemental files; https://doi.org/10.1536/ihj.17-672 